\title{
Atypical Type A Thymoma
}

National Cancer Institute

\section{Source}

National Cancer Institute. Atypical Type A Thymoma. NCI Thesaurus. Code C146640.

A rare type A thymoma displaying atypical features (hypercellularity, increased mitotic activity, and focal necrosis). 